\title{
Genetic Characterization of Methicillin Resistant and Sensitive, Vancomycin Intermediate Staphylococcus aureus Strains Isolated from Different Iranian Hospitals
}

\author{
Seyed Asghar Havaei, ${ }^{1}$ Amir Azimian, ${ }^{1}$ Hosein Fazeli,, ${ }^{1}$ Mahmood Naderi, ${ }^{2,3}$ \\ Kiarash Ghazvini, ${ }^{4}$ Siamak Mirab Samiee, ${ }^{5,6}$ Zahra Masoumi, $^{3}$ and Mojtaba Akbari ${ }^{7}$ \\ ${ }^{1}$ Department of Microbiology, School of Medicine, Isfahan University of Medical Sciences, P.O. Box 73461-8174, Isfahan, Iran \\ ${ }^{2}$ Department of Medical Biotechnology, School of Medical Sciences, Tarbiat Modares University, Tehran, Iran \\ ${ }^{3}$ Department of Molecular Biology and Genetic Engineering, Stem Cell Technology Research Center, Tehran, Iran \\ ${ }^{4}$ Department of Microbiology, School of Medicine, Mashhad University of Medical Sciences, Mashhad, Iran \\ ${ }^{5}$ Food and Drug Laboratory Research Center, Ministry of Health and Medical Education, No. 408, Emam Khomeini Avenue, \\ Tehran, Iran \\ ${ }^{6}$ Reference Health Laboratories, Ministry of Health and Medical Education, Tehran, Iran \\ ${ }^{7}$ Department of Epidemiology, School of Medicine, Isfahan University of Medical Sciences, P.O. Box 73461-8174, Isfahan, Iran
}

Correspondence should be addressed to Amir Azimian, amir_azimian2003@yahoo.com

Received 5 November 2012; Accepted 22 November 2012

Academic Editors: C. K. Cote, P. Di Martino, S. H. Flint, and G. Koraimann

Copyright $\odot 2012$ Seyed Asghar Havaei et al. This is an open access article distributed under the Creative Commons Attribution License, which permits unrestricted use, distribution, and reproduction in any medium, provided the original work is properly cited.

\begin{abstract}
Background. Global concerns have been raised due to upward trend of Vancomycin Intermediate Staphylococcus aureus (VISA) and Vancomycin Resistant Staphylococcus aureus (VRSA) reports which mean casting doubt on the absolute effectiveness of the last line of antibiotic treatment for $S$. aureus, vancomycin. Hence, epidemiological evaluation can improve global health care policies. Methodology. 171 Isolates of Staphylococcus aureus were collected from different types of clinical samples in selected hospitals in Isfahan, Mashhad, and Tehran, Iran. Then, they were evaluated by agar screening, disk diffusion, and MIC method to determine their resistance to vancomycin and methicillin. The isolated VISA strains were then confirmed with genetic analysis by the evaluation of mecA and vanA genes, SCCmec, agr, and spa type, and also toxin profiles. MLST was also performed. Results and Conclusion. Our data indicated that $67 \%$ of isolated S. aureus strains were resistant to methicillin. Furthermore, five isolates $(2.9 \%)$ had intermediate resistance to vancomycin (VISA). In contrast to usual association of VISA with MRSA strains, we found two isolates of MSSA-VISA. Therefore, our data suggests a probable parallel growing trend of VISA towards MSSA, along with MRSA strains. However, more samples are required to confirm these primarily data. Moreover, genetic analysis of the isolated VISA strains revealed that these strains are endemic Asian clones.
\end{abstract}

\section{Introduction}

Staphylococcus aureus is one of the major causes of hospital and community acquired infections that can vary from mild superficial lesions to acute deep and systemic infections. Increasing antimicrobial resistance in Staphylococcus aureus has led to major concerns about the future of antimicrobial therapy of its infections. Finding methicillin resistant Staphylococcus aureus (MRSA) strains, just two years after the clinical use of betalactamase resistant betalactams, put specialists into trouble with treating these infections. About twenty years later in 1980s, alternative effective anti-Staphylococcal antibiotics such as vancomycin were introduced $[1,2]$. Thence, for many years vancomycin was the drug of choice for MRSA infections, until the first $S$. aureus isolate with reduced sensitivity to vancomycin (vancomycin intermediate Staphylococcus aureus (VISA)) was reported in Japan in 1997 [3]. Thereafter, several reports of VISA from USA [4], France [5], Brazil [6], Korea [7], and other parts of the world [8] were published and resulted in increasing concerns 
about the effectiveness of vancomycin therapy in serious Staphylococcal infections. Reduced sensitivity to vancomycin in $S$. aureus occurs due to several genetic and phenotypic alterations in wild-type bacteria including altered expression of regulatory genetic elements, thickness of cell wall, changes in the penicillin binding protein (PBP) profiles, and decreased cell wall autolysis [9]. Since 2002, there have been also several reports of vancomycin resistant Staphylococcus aureus (VRSA) strains, mainly from the United States, India, and Iran [10-19]. The majority of the reported VISA isolates are MRSA strains with reduced susceptibility to vancomycin even though a few reports have also shown this phenomenon in methicillin sensitive Staphylococcus aureus (MSSA) isolates [9, 20-22]. Data obtained from genetic analysis of VISA isolates was rarely reported in Asian countries, particularly from Iran. Here, we reported genetic characteristics of five VISA strains, isolated from admitted patients in three teaching hospitals in Isfahan, Mashhad, and Tehran, Iran, in the autumn of 2011.

\section{Methodology}

171 isolates of Staphylococcus aureus were obtained from patients admitted between September 232011 and December 212011 at selected hospitals in Isfahan, Mashhad, and Tehran, Iran.

These isolates were taken from blood, urine, sputum, wound, abscess, nose, throat, eye, and respiratory tract samples. S. aureus isolates were identified by conventional biochemical tests including gram staining, catalase, mannitol fermentation, slide, and tube coagulase test and DNase.

2.1. Screening for Methicillin and Vancomycin Resistance. All S. aureus isolates were screened for oxacillin and vancomycin resistance using agar screening method. The isolates that had grown in vancomycin agar screening media were retested with phenotypic tests to confirm the identification. It is noteworthy that the data obtained from spa typing, considered to be highly specific for $S$. aureus, was in align with phenotypic data and confirmed the isolated strains as $S$. aureus.

2.2. Antimicrobial Susceptibility Test. Antibiotic susceptibility test was carried out using disk diffusion method (MAST DISKS) according to guidelines of Clinical Laboratory Standards Institute (CLSI) $[23,24]$. The list of the antibiotics used in the test is presented in Table 2. Strain of S. aureus ATCC 25923 was used as control.

2.3. MIC Determination. Oxacillin and vancomycin agar dilution was used for all $S$. aureus isolates according to guidelines of Clinical Laboratory Standards Institute (CLSI). $E$-Test method (Biomeriux Strips) was used for redetermination of MIC results for $S$. aureus strains capable of growing in the vancomycin agar screening plate and/or having vancomycin MIC $4-8 \mu \mathrm{g} / \mathrm{mL}$. Methicillin resistance was defined as the capability of growth in agar screening media including $4 \% \mathrm{NaCl}+6 \mu \mathrm{g} / \mathrm{mL}$ oxacillin and MIC $\geq 4 \mu \mathrm{g} / \mathrm{mL}$ and vancomycin intermediate resistance was defined as vancomycin MIC of $4-8 \mu \mathrm{g} / \mathrm{mL}$ [23]. Strains of Staphylococcus aureus ATCC 29213 and Enterococcus faecalis ATCC 52199 were used as controls.

2.4. Genomic DNA Extraction. Genomic DNAs of S. aureus isolates were extracted using QIAamp DNA minikit. According to manufacturer's protocol for bacterial cells, we added lysostaphin at the final concentration of $30 \mu \mathrm{g} / \mathrm{mL}$ in lysis buffer.

2.5. PCR. PCR amplification was performed with a TAKARA Gradient PCR TP600 thermal cycler in a volume of $50 \mu \mathrm{L}$. We used EmeraldAmp MAX PCR Master Mix (Takara, Japan) for all PCR reactions.

(i) PCR Identification of mecA and vanA Genes. The primers used for amplification of mecA and vanA genes are listed in Table 3. PCR was performed with the following thermal setting: $5 \mathrm{~min}$ at $94^{\circ} \mathrm{C}$ for initial enzyme activation followed by 40 cycles of amplification (denaturation at $94^{\circ} \mathrm{C}$ for $30 \mathrm{sec}$ for $m e c A$ and $1 \mathrm{~min}$ for vanA; annealing at $57^{\circ} \mathrm{C}$ for $45 \mathrm{sec}$ for $m e c A$ and at $55^{\circ} \mathrm{C}$ for $1 \mathrm{~min}$ for $v a n A$; extension at $72^{\circ} \mathrm{C}$ for $30 \mathrm{sec}$ for $\operatorname{mec} A$ and $2 \mathrm{~min}$ for $v a n A$ ) and final extension at $72^{\circ} \mathrm{C}$ for $5 \mathrm{~min}$.

(ii) Multiplex PCR for Detection of Toxin Genes. The primers used for amplification of Panton-Valentine Leukocidin ( $p v l$ ), Toxic Shock Syndrome Toxin-1 (tst1), alpha hemolysin (hla), and Enterotoxin $C(\mathrm{sec})$ genes are listed in Table 3. PCR was performed with the following thermal setting: $5 \mathrm{~min}$ at $94^{\circ} \mathrm{C}$ for initial enzyme activation followed by 40 cycles of amplification (denaturation at $94^{\circ} \mathrm{C}$ for $40 \mathrm{sec}$; annealing at $60^{\circ} \mathrm{C}$ for $40 \mathrm{sec}$; extension at $72^{\circ} \mathrm{C}$ for $1 \mathrm{~min}$ ) and final extension at $72^{\circ} \mathrm{C}$ for $5 \mathrm{~min}$.

(iii) Multiplex PCR for SCCmec and agr Typing. SCCmec and agr typing were performed as previously described $[25,26]$. The primers used for the PCR are listed in Table 3.

2.6. MLST. Multilocus Sequence Typing was carried out by PCR and sequencing of the internal fragments of arc, aro, $g l p$, gmk, pta, tpi, and yqi genes of S. aureus as previously described [27].

2.7. spa Typing. Determination of spa type was performed by PCR and sequencing of polymorphic X region of spa gene was carried out as previously described [28].

2.8. Nucleotide Sequencing. Amplified PCR products were purified with QIAquick Gel Extraction Kit. The purified PCR products were sequenced with an ABI 3730XL DNA analyzer (Applied Biosystems) in both directions. The sequences were used for both confirmation and sequence-based typing methods (MLST and spa typing).

\section{Results}

Of $171 \mathrm{~S}$. aureus isolates analyzed, we found that 115 isolates $(67 \%)$ were MRSA and 5 isolates (2.9\%) showed 
TABLE 1: Genetic characteristics of VISA isolates.

\begin{tabular}{lccccccccccccc}
\hline & agr & Sccmec & pvl & hla & etc & ts1t & mecA & vanA & spa type & spa repeat profile & ST & CC & MLST allelic profile \\
\hline VISA1 & I & III & + & + & + & + & + & - & t037 & $15-12-16-02-25-17-24$ & ST-1283 & 8 & $2-3-1-88-4-4-3$ \\
VISA2 & I & I & - & + & - & - & + & - & t037 & $15-12-16-02-25-17-24$ & ST-585 & 8 & $2-1-1-1-4-4-3$ \\
VISA3 & I & III & - & + & - & - & + & - & t037 & $15-12-16-02-25-17-24$ & ST-239 & 8 & $2-3-1-1-4-4-3$ \\
VISA4 & II & - & + & + & - & - & - & - & t008 & $11-19-12-21-17-34-24-34-22-25$ & ST-8 & 8 & $3-3-1-1-4-4-3$ \\
VISA5 & II & - & - & - & - & + & - & - & t0189 & $07-23-12-21-17-34$ & ST-8 & 8 & $3-3-1-1-4-4-3$ \\
\hline
\end{tabular}

pvl: Panton-Valentin Leukocidin, sec: Enterotoxin C, tst1: Toxic Shock Syndrome Toxin1, hla: alpha Hemolysin, agr: accessory gene regulator, SCCmec: Staphylococcal Cassette Chromosome, spa: Staphylococcal protein A.

TABLE 2: Phenotypic characteristics of VISA isolates.

\begin{tabular}{lllllllllllllllll}
\hline \multicolumn{3}{c}{ Gender/age } & city & Sample/ward & Oxa & Van & Min & Lev & Cip & Tet & Cot & Gen & Cli & Rif & Oxa MIC Van MIC Van Agar screen \\
\hline VISA1 & M/15 & Mashhad & Blood/neurology & R & S & S & S & S & R & S & R & S & S & 128 & 8 & + \\
VISA2 & M/39 & Isfahan & Throat/out patient & R & S & S & R & R & R & R & R & R & R & 128 & 4 \\
VISA3 & M/50 & Isfahan & Blood/neurology & R & S & R & S & S & S & S & S & R & R & 64 & 4 & + \\
VISA4 & M/52 & Mashhad & Wound/urgent & S & S & R & S & S & S & S & S & R & S & 2 & 4 & + \\
VISA5 & M/71 & Mashhad & Throat/Internal & S & S & I & R & R & R & R & S & R & I & 2 & 4 \\
\hline
\end{tabular}

Oxa: oxacillin, Van: vancomycin, Min: minocyclin, Lev: levofloxacin, Cip: ciprofloxacin, Tet: tetracycline, Cot: cotrimoxazole, Gen: gentamycin, Cli: clindamycin, Rif: rifampicin, R: resistant, S: susceptible.

intermediate resistance to vancomycin. The demographic data and antibiogram results of these strains are listed in Table 2. Moreover, genetic evaluation results including mecA and vanA genes PCR, Sccmec types, agr groups, toxin profiles, spa types and Sequence Types (STs) are presented in Table 1.

\section{Discussion}

Isolation of VISA strains has been reported in many parts of the world. Resistance to vancomycin has led to global concerns owing to the fact that vancomycin is considered as the last effective drug of choice for Staphylococcal infections [3-8]. The isolation of heteroresistant VISA (hVISA) and VISA strains has been increasingly reported from different Asian countries with various prevalence rates $[3,7,29,30]$. There are not many reports concerning the prevalence rate of VISA strains in Iran; however, in 2008, Saderi et al. reported the mean prevalence rate of $1.8 \%$ for VISA isolated from clinical samples based on evaluation of four teaching hospitals in Tehran [31]. In our study, we found five VISA strains $(2.9 \%)$ amongst $171 \mathrm{~S}$. aureus isolates. Our data shows an upward trend in the isolation of VISA strains in Iran that might be due to the high and uncontrolled vancomycin prescription rate, even though more research is needed to elucidate the matter.

Some reports have been published concerning the antimicrobial susceptibility patterns of VISA strains, isolated in various parts of the world $[32,33]$. In most of these studies, the majority of the isolates were resistant to clindamycin but were susceptible to most of the other anti-Staphylococcal antibiotics [34-36]. Most of VISA strains in this study were also resistant to clindamycin. In contrast, Levofloxacin, Ciprofloxacin, Cotrimoxazole, and Gentamycin were effective on most of the VISA isolates (Table 2).

The majority of the previously reported VISA strains were resistant to methicillin; however, there have been also a few reports about methicillin sensitive Staphylococcus aureusVISA (MSSA-VISA) strains [9]. Two isolates of our five VISA strains, both obtained from the same hospital in Mashhad, were susceptible to methicillin; however, one of them was isolated from throat sample of a hospitalized patient in internal ward and another was isolated from wound sample of an outpatient in urgent ward that had not had any history of hospitalization. With regard to genetic characteristics, the two strains were similar; for instance, both of them belonged to agr group II and sequence type 8 (ST8) and therefore, clonal complex 8 (CC8). However, their spa types and toxin profiles were different. The wound isolated strain (VISA4) had spa type t008 and was positive for Panton-Valentine Leukocidin ( $p v l$ ) and alpha Hemolysin (hla) genes. On the other hand, the throat isolated strain (VISA5) belonged to spa type t0189 and merely had Toxic Shock Syndrome Toxin (tst1) gene. Owing to the fact that sequence type is considered as a long-term and stable epidemiological marker over time and due to full homology in the sequence type of the two isolates, we deduced that they might have a common ancestor. On the other hand, alterations in some genes, such as spa and virulence factor genes, might be due to the fact that these elements are more prone to genetic alterations in a shorter period of time.

The relation between agr group, type of infection, geographical area, and resistance to some antibiotics, particularly vancomycin, is highlighted in some articles [37]. Impaired function of accessory gene regulator (agr) has been associated with the development of VISA strains [9]. agr I or II is associated with VISA strains isolated from diverse geographical regions $[32,33,37,38]$. Our findings are in concordance with these reports. Both of our MSSA-VISA strains belonged to agr II but the other three MRSA-VISA strains had agr I.

All the patients with MRSA-VISA either had a previous history of hospitalization or were being hospitalized at the 
Table 3: Primers used in this study.

\begin{tabular}{|c|c|c|c|c|}
\hline Target & Primer & Sequence $\left(5^{\prime}-3^{\prime}\right)$ & Product size (bp) & Reference \\
\hline \multirow{2}{*}{ mecA } & $\mathrm{F}$ & AGAAGATGGTATGTGGAAGTTAG & \multirow{2}{*}{583} & \multirow{2}{*}{ This study } \\
\hline & $\mathrm{R}$ & ATGTATGTGCGATTGTATTGC & & \\
\hline \multirow{2}{*}{$p v l$} & $\mathrm{~F}$ & GGAAACATTTATTCTGGCTATAC & \multirow{2}{*}{502} & \multirow{2}{*}{ This study } \\
\hline & $\mathrm{R}$ & CTGGATTGAAGTTACCTCTGG & & \\
\hline \multirow{2}{*}{ hla } & $\mathrm{F}$ & CGGTACTACAGATATTGGAAGC & \multirow{2}{*}{744} & \multirow{2}{*}{ This study } \\
\hline & $\mathrm{R}$ & TGGTAATCATCACGAACTCG & & \\
\hline \multirow{2}{*}{$\sec$} & $\mathrm{F}$ & GGGAATGTTGGATGAAGG & \multirow{2}{*}{900} & \multirow{2}{*}{ This study } \\
\hline & $\mathrm{R}$ & AGGCAAGCACCGAAGTAC & & \\
\hline \multirow{2}{*}{ tst1 } & $\mathrm{F}$ & TTATCGTAAGCCCTTTGTTG & \multirow{2}{*}{398} & \multirow{2}{*}{ This study } \\
\hline & $\mathrm{R}$ & TAAAGGTAGTTCTATTGGAGTAGG & & \\
\hline \multirow{5}{*}{$a g r$} & Pan F & ATGCACATGGTGCACATGC & & \multirow{5}{*}[26]{} \\
\hline & R I & GTCACAAGTACTATAAGCTGCGAT & 439 & \\
\hline & R II & GTATTACTAATTGAAAAGTGCCATAGC & 572 & \\
\hline & R III & CTGTTGAAAAAGTCAACTAAAAGCTC & 406 & \\
\hline & R IV & CGATAATGCCGTAATACCCG & 657 & \\
\hline \multirow{8}{*}{ SCCmec } & $\mathrm{F} \beta$ & ATTGCCTTGATAATAGCCYTCT & 937 & \multirow{8}{*}[25]{} \\
\hline & $\mathrm{R} \alpha 3$ & TAAAGGCATCAATGCACAAACACT & & \\
\hline & F ccrC & CGTCTATTACAAGATGTTAAGGATAAT & 518 & \\
\hline & $\mathrm{R} \operatorname{ccrC}$ & CCTTTATAGACTGGATTATTCAAAATAT & \multirow{3}{*}{415} & \\
\hline & F 1272 & GCCACTCATAACATATGGAA & & \\
\hline & R 1272 & CATCCGAGTGAAACCCAAA & & \\
\hline & F $5 \mathrm{R} m e c A$ & TATACCAAACCCGACAACTAC & \multirow[t]{2}{*}{359} & \\
\hline & R 5R431 & CGGCTACAGTGATAACATCC & & \\
\hline
\end{tabular}

time of sampling. Moreover, their SCCmec types belonged to types I and III. Collectively, the data indicates that the isolated MRSA-VISA strains were hospital-acquired MRSA (HA-MRSA). VISA1 strain was positive for all evaluated toxin genes including $p v l$, hla, sec, and tst 1 which can increase its potential pathogenicity; however, this isolate was susceptible to most of the tested antibiotic disks (Table 2). Interestingly, $p v l$ gene was found in HA-MRSA "VISA1" despite the fact that it is mostly found in CA-MRSA strains. VISA 2, 3 did not have any major toxin genes.

All of our MRSA-VISA isolates (two isolates from Isfahan in center of Iran and one isolate from Mashhad in northeast of Iran) had spa t037 and their STs were ST1283, ST585, and ST239 for VISA 1, 2, and 3, respectively. ST1283 and ST585 are single-locus variants (SLV) of ST239, the endemic strain of Asia (Figure 1) which our data suggest to be the probable common ancestor of our MRSA-VISA isolates. All of our five VISA isolates belonged to clonal complex 8 (CC8) that is the major endemic S. aureus clone (particularly MRSA) in Asia and also is one of the pandemic MRSA clones [33, 39-44].

Increasing antibiotic resistance in major $S$. aureus clones intensifies precautionary policies for public health care systems. In this study, we have shown the upward trend of MSSA- and MRSA-VISA clones in Iran. Moreover, findings of our study suggest that the isolated VISA strains most probably belong to endemic Asian clones. In addition, these data suggest that vancomycin resistance has the potential to

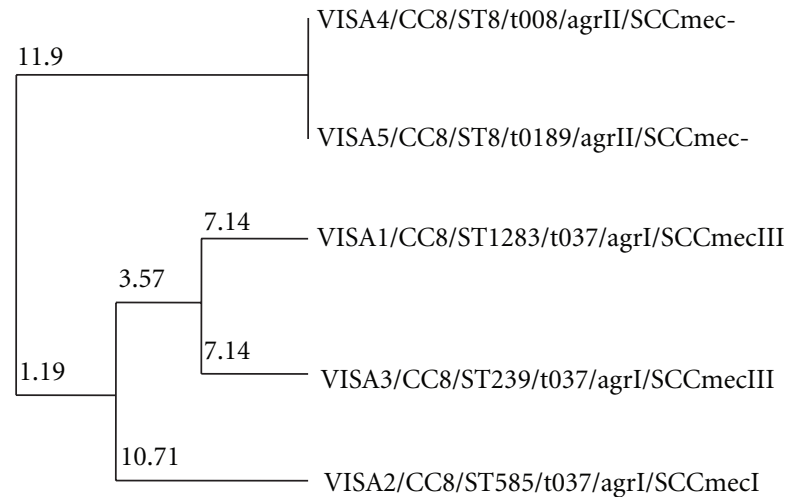

FIGURE 1: Dendrogram comparing sequence types (STs) of VISA isolates.

become a widespread problem in both MRSA and MSSA strains.

\section{Conflict of Interests}

S. A. Havaei, A. Azimian, H. Fazeli, M. Naderi, K. Ghazvini, S. M. Samiee, and Z. Masoumi have no conflict of interests.

\section{Acknowledgment}

The authors particularly thank Dr. Jean B. Patel from the Division of Healthcare Quality Promotion, Centers 
for Disease Control, for her scientific advice. The authors also thank Stem Cell Research Center (Bone Yakhteh) for supporting technical assistance. The authors deeply thank Professor Richard P. Novick and Professor K. Hiramatsu for providing standard strains. The authors thank Dr. Mehdi Paryan for assistance in collecting clinical isolates of $S$. aureus, Dr. Ehsan Arefian, Dr. Bahram Nasr Esfahani, and Dr. Sharareh Moghim for their scientific advice. This work was supported by the Isfahan University of Medical Sciences (Grant no. 390256).

\section{References}

[1] S. J. Rehm and A. Tice, "Staphylococcus aureus: methicillinsusceptible $S$. aureus to methicillin-resistant $S$. aureus and vancomycin-resistant S. aureus," Clinical Infectious Diseases, vol. 51, no. 2, pp. S176-S182, 2010.

[2] D. M. Sievert, J. T. Rudrik, J. B. Patel, L. C. McDonald, M. J. Wilkins, and J. C. Hageman, "Vancomycin-resistant Staphylococcus aureus in the United States, 2002-2006," Clinical Infectious Diseases, vol. 46, no. 5, pp. 668-674, 2008.

[3] K. Hiramatsu, H. Hanaki, T. Ino, K. Yabuta, T. Oguri, and F. C. Tenover, "Methicillin-resistant Staphylococcus aureus clinical strain with reduced vancomycin susceptibility," Journal of Antimicrobial Chemotherapy, vol. 40, no. 1, pp. 135-136, 1997.

[4] T. R. Walsh and R. A. Howe, "The prevalence and mechanisms of vancomycin resistance in Staphylococcus aureus," Annual Review of Microbiology, vol. 56, pp. 657-675, 2002.

[5] M. C. Ploy, C. Grélaud, C. Martin, L. De Lumley, and F. Denis, "First clinical isolate of vancomycin-intermediate Staphylococcus aureus in a French hospital," Lancet, vol. 351, no. 9110, article 1212, 1998.

[6] G. A. Oliveira, A. M. Dell'Aquila, R. L. Masiero et al., "Isolation in Brazil of nosocomial Staphylococcus aureus With reduced susceptibility to vancomycin," Infection Control and Hospital Epidemiology, vol. 22, no. 7, pp. 443-448, 2001.

[7] M.-N. Kim, C. H. Pai, J. H. Woo, J. S. Ryu, and K. Hiramatsu, "Vancomycin-intermediate Staphylococcus aureus in Korea," Journal of Clinical Microbiology, vol. 38, no. 10, pp. 3879-3881, 2000.

[8] S. K. Fridkin, "Vancomycin-intermediate and -resistant Staphylococcus aureus: what the infectious disease specialist needs to know," Clinical Infectious Diseases, vol. 32, no. 1, pp. 108-115, 2001.

[9] S. K. Pillai, C. Wennersten, L. Venkataraman, G. M. Eliopoulos, R. C. Moellering, and A. W. Karchmer, "Development of reduced vancomycin susceptibility in methicillin-susceptible Staphylococcus aureus," Clinical Infectious Diseases, vol. 49, no. 8, pp. 1169-1174, 2009.

[10] M. Aligholi, M. Emaneini, F. Jabalameli, S. Shahsavan, H. Dabiri, and H. Sedaght, "Emergence of high-level vancomycin-resistant Staphylococcus aureus in the Imam Khomeini hospital in Tehran," Medical Principles and Practice, vol. 17, no. 5, pp. 432-434, 2008.

[11] CDC, "Public Health Dispatch: vancomycin-resistant Staphylococcus aureus-Pennsylvania," Morbidity and Mortality Weekly Report, vol. 51, no. 40, p. 902, 2002.

[12] S. Chang, D. M. Sievert, J. C. Hageman et al., "Infection with vancomycin-resistant Staphylococcus aureus containing the vanA resistance gene," New England Journal of Medicine, vol. 348, no. 14, pp. 1342-1347, 2003.
[13] A. Dezfulian, M. M. Aslani, M. Oskoui et al., "Identification and characterization of a high vancomycin-resistant Staphylococcus aureus harboring VanA gene cluster isolated from diabetic foot ulcer," Iranian Journal of Basic Medical Sciences, vol. 15, no. 2, pp. 803-806, 2012.

[14] M. Kacica and L. McDonald, "Brief Report: vancomycinresistant Staphylococcus aureus-New York," Morbidity and Mortality Weekly Report, vol. 53, no. 15, pp. 522-523, 2004.

[15] D. Miller, V. Urdaneta, A. Weltman, and S. Park, "Vancomycin-resistant Staphylococcus aureus," Morbidity and Mortality Weekly Report, vol. 51, p. 902, 2002.

[16] B. Saha, A. K. Singh, A. Ghosh, and M. Bal, "Identification and characterization of a vancomycin-resistant Staphylococcus aureus isolated from Kolkata (South Asia)," Journal of Medical Microbiology, vol. 57, no. 1, pp. 72-79, 2008.

[17] V. Thati, C. T. Shivannavar, and S. M. Gaddad, "Vancomycin resistance among methicillin resistant Staphylococcus aureus isolates from intensive care units of tertiary care hospitals in Hyderabad," Indian Journal of Medical Research, vol. 134, no. 5, pp. 704-708, 2011.

[18] H. K. Tiwari and M. R. Sen, "Emergence of vancomycin resistant Staphylococcus aureus (VRSA) from a tertiary care hospital from northern part of India," BMC Infectious Diseases, vol. 6, article 156, 2006.

[19] L. M. Weigel, R. M. Donlan, D. H. Shin et al., "High-level vancomycin-resistant Staphylococcus aureus isolates associated with a polymicrobial biofilm," Antimicrobial Agents and Chemotherapy, vol. 51, no. 1, pp. 231-238, 2007.

[20] T. S. Naimi, D. Anderson, C. O’Boyle et al., "Vancomycinintermediate Staphylococcus aureus with phenotypic susceptibility to methicillin in a patient with recurrent bacteremia," Clinical Infectious Diseases, vol. 36, no. 12, pp. 1609-1612, 2003.

[21] J. C. Hageman, D. A. Pegues, C. Jepson et al., "Vancomycinintermediate Staphylococcus aureus in a home health-care patient," Emerging Infectious Diseases, vol. 7, no. 6, pp. 10231025, 2001.

[22] S. Bobin-Dubreux, M. E. Reverdy, C. Nervi et al., "Clinical isolate of vancomycin-heterointermediate Staphylococcus aureus susceptible to methicillin and in vitro selection of a vancomycin-resistant derivative," Antimicrobial Agents and Chemotherapy, vol. 45, no. 1, pp. 349-352, 2001.

[23] P. A. Wayne, "Performance standards for antimicrobial susceptibility testing," CLSI document M100-S20, 20th informational supplement. Clinical Laboratory Standard Institute, 2010.

[24] P. A. Wayne, "Performance standards for antimicrobial disk susceptibility tests," CLSI document M02-A11, 11th ed. Clinical Laboratory Standard Institute, 2012.

[25] K. Boye, M. D. Bartels, I. S. Andersen, and J. A. Møller, "A new multiplex PCR for easy screening of methicillin-resistant Staphylococcus aureus SCCmec types I-V," Clinical Microbiology and Infection, vol. 13, no. 7, pp. 725-727, 2007.

[26] S. N. Peerayeh, A. Azimian, Q. B. Nejad, and M. Kashi, "Prevalence of agr specificity groups among Staphylococcus aureus isolates from university hospitals in Tehran," Laboratory Medicine, vol. 40, no. 1, pp. 27-29, 2009.

[27] M. C. Enright, N. P. J. Day, C. E. Davies, S. J. Peacock, and B. G. Spratt, "Multilocus sequence typing for characterization of methicillin-resistant and methicillin-susceptible clones of Staphylococcus aureus," Journal of Clinical Microbiology, vol. 38, no. 3, pp. 1008-1015, 2000.

[28] D. Harmsen, H. Claus, W. Witte et al., "Typing of methicillinresistant Staphylococcus aureus in a University hospital setting 
by using novel software for spa repeat determination and database management," Journal of Clinical Microbiology, vol. 41, no. 12, pp. 5442-5448, 2003.

[29] S.-Y. Lin, T.-C. Chen, F.-J. Chen et al., "Molecular epidemiology and clinical characteristics of hetero-resistant vancomycin intermediate Staphylococcus aureus bacteremia in a Taiwan Medical Center," Journal of Microbiology, Immunology and Infection, vol. 45, no. 6, pp. 435-441, 2012.

[30] J. H. Song, K. Hiramatsu, Y. S. Ji et al., "Emergence in Asian countries of Staphylococcus aureus with reduced susceptibility to vancomycin," Antimicrobial Agents and Chemotherapy, vol. 48, no. 12, pp. 4926-4928, 2004.

[31] H. Saderi, P. Owlia, Z. Maleki, M. Habibi, and N. Rahmati, "Susceptibility to vancomycin in Staphylococcus aureus isolated from patients of four university-affiliated hospitals in Tehran," Iran Journal of Pathology, vol. 3, no. 3, pp. 161-166, 2008.

[32] P. R. Hsueh, S. Y. Lee, C. L. Perng, T. Y. Chang, and J. J. Lu, "Clonal dissemination of meticillin-resistant and vancomycinintermediate Staphylococcus aureus in a Taiwanese hospital," International Journal of Antimicrobial Agents, vol. 36, no. 4, pp. 307-312, 2010.

[33] R. A. Howe, A. Monk, M. Wootton, T. R. Walsh, and M. C. Enright, "Vancomycin susceptibility within methicillinresistant Staphylococcus aureus lineages," Emerging Infectious Diseases, vol. 10, no. 5, pp. 855-857, 2004.

[34] S. K. Fridkin, J. Hageman, L. K. McDougal et al., "Epidemiological and microbiological characterization of infections caused by Staphylococcus aureus with reduced susceptibility to vancomycin, United States, 1997-2001," Clinical Infectious Diseases, vol. 36, no. 4, pp. 429-439, 2003.

[35] C. J. Graber, M. K. Wong, H. A. Carleton, F. Perdreau-Remington, B. L. Haller, and H. F. Chambers, "Intermediate vancomycin susceptibility in a community-associated MRSA clone," Emerging Infectious Diseases, vol. 13, no. 3, pp. 491-493, 2007.

[36] L. D. Saravolatz, J. Pawlak, L. B. Johnson, L. D. Saravolatz, and N. Husain, "In vitro activity of ceftobiprole against meticillin-resistant Staphylococcus aureus (MRSA), vancomycin-intermediate $S$. aureus (VISA), vancomycinresistant $S$. aureus (VRSA) and daptomycin-non-susceptible S. aureus (DNSSA)," International Journal of Antimicrobial Agents, vol. 36, no. 5, pp. 478-480, 2010.

[37] G. Sakoulas, G. M. Eliopoulos, R. C. Moellering et al., "Accessory gene regulator (agr) locus in geographically diverse Staphylococcus aureus isolates with reduced susceptibility to vancomycin," Antimicrobial Agents and Chemotherapy, vol. 46, no. 5, pp. 1492-1502, 2002.

[38] I. Verdier, M. E. Reverdy, J. Etienne, G. Lina, M. Bes, and F. Vandenesch, "Staphylococcus aureus isolates with reduced susceptibility to glycopeptides belong to accessory gene regulator group I or II," Antimicrobial Agents and Chemotherapy, vol. 48, no. 3, pp. 1024-1027, 2004.

[39] S. Shahsavan, L. Jabalameli, P. Maleknejad et al., "Molecular analysis and antimicrobial susceptibility of methicillin resistant Staphylococcus aureus in one of the hospitals of Tehran University of Medical Sciences: high prevalence of sequence type 239 (ST239) clone," Acta Microbiologica et Immunologica Hungarica, vol. 58, no. 1, pp. 31-39, 2011.

[40] K. S. Ko, J. Y. Lee, Y. S. Ji et al., "Distribution of major genotypes among methicillin-resistant Staphylococcus aureus clones in Asian countries," Journal of Clinical Microbiology, vol. 43, no. 1, pp. 421-426, 2005.

[41] H. Chen, Y. Liu, X. Jiang, M. Chen, and H. Wang, "Rapid change of methicillin-resistant Staphylococcus aureus clones in a Chinese Tertiary Care Hospital over a 15-year period," Antimicrobial Agents and Chemotherapy, vol. 54, no. 5, pp. 1842-1847, 2010.

[42] H. Y. Cha, H. O. Kim, J. S. Jin, and J. C. Lee, "Emergence of vancomycin-intermediate Staphylococcus aureus from predominant methicillin-resistant $S$. aureus clones in a Korean hospital," Journal of Microbiology, vol. 48, no. 4, pp. 533-535, 2010.

[43] W. Y. Wang, S. Y. Lee, T. S. Chiueh, and J. J. Lu, "Molecular and phenotypic characteristics of methicillin-resistant and vancomycin-intermediate Staphylococcus aureus isolates from patients with septic arthritis," Journal of Clinical Microbiology, vol. 47, no. 11, pp. 3617-3623, 2009.

[44] L. K. McDougal, C. D. Steward, G. E. Killgore, J. M. Chaitram, S. K. McAllister, and F. C. Tenover, "Pulsed-field gel electrophoresis typing of oxacillin-resistant Staphylococcus aureus isolates from the United States: establishing a national database," Journal of Clinical Microbiology, vol. 41, no. 11, pp. 5113-5120, 2003. 

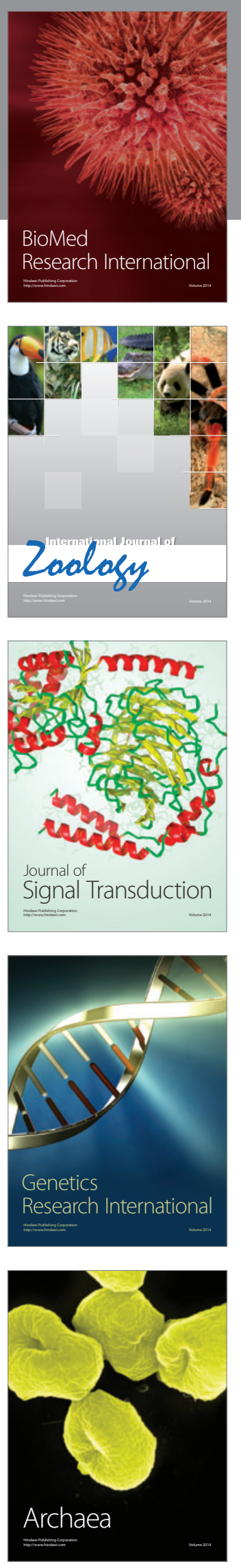
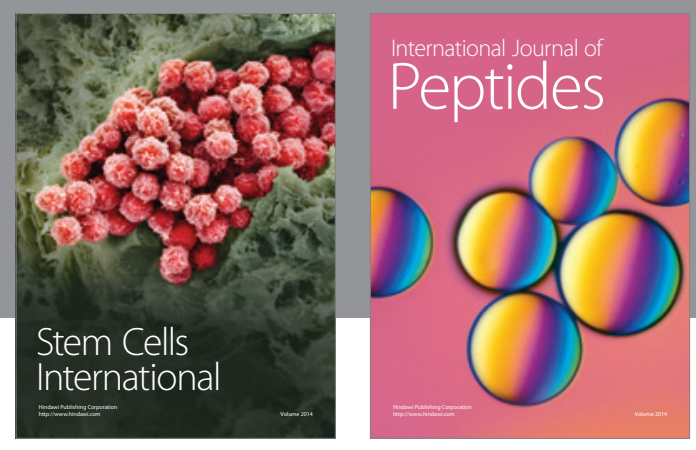

Submit your manuscripts at

http://www.hindawi.com
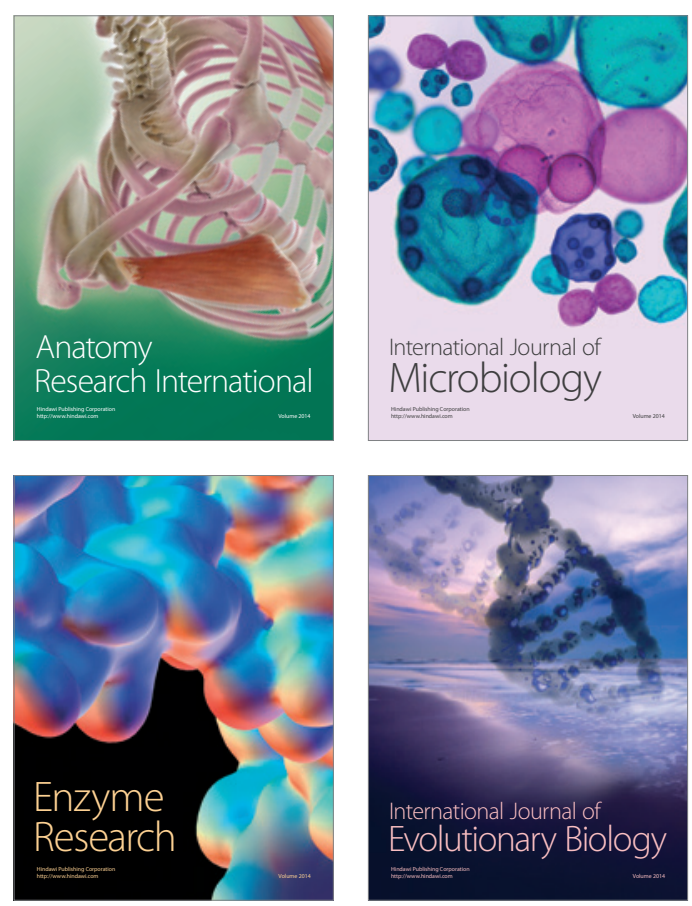
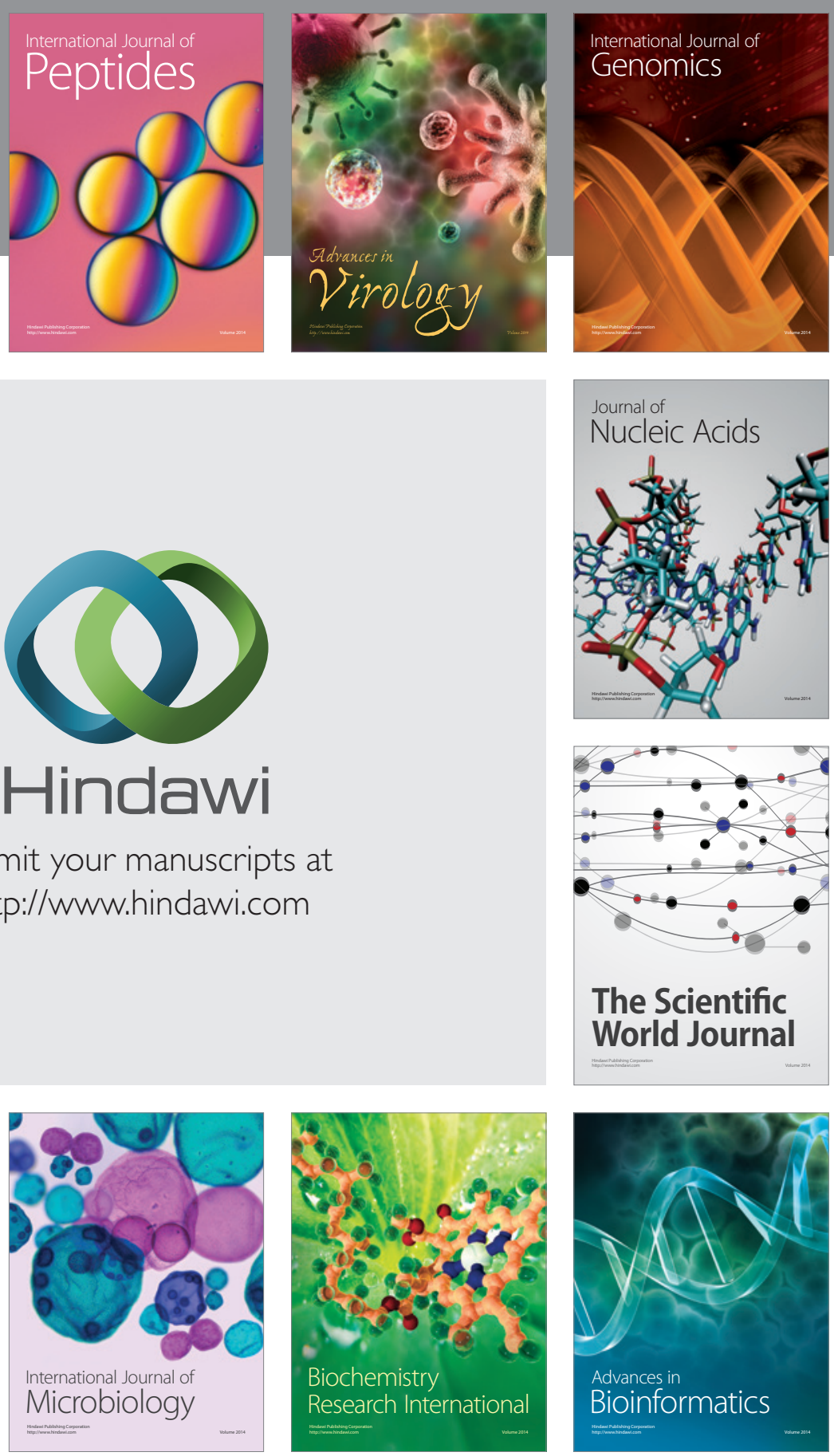

The Scientific World Journal
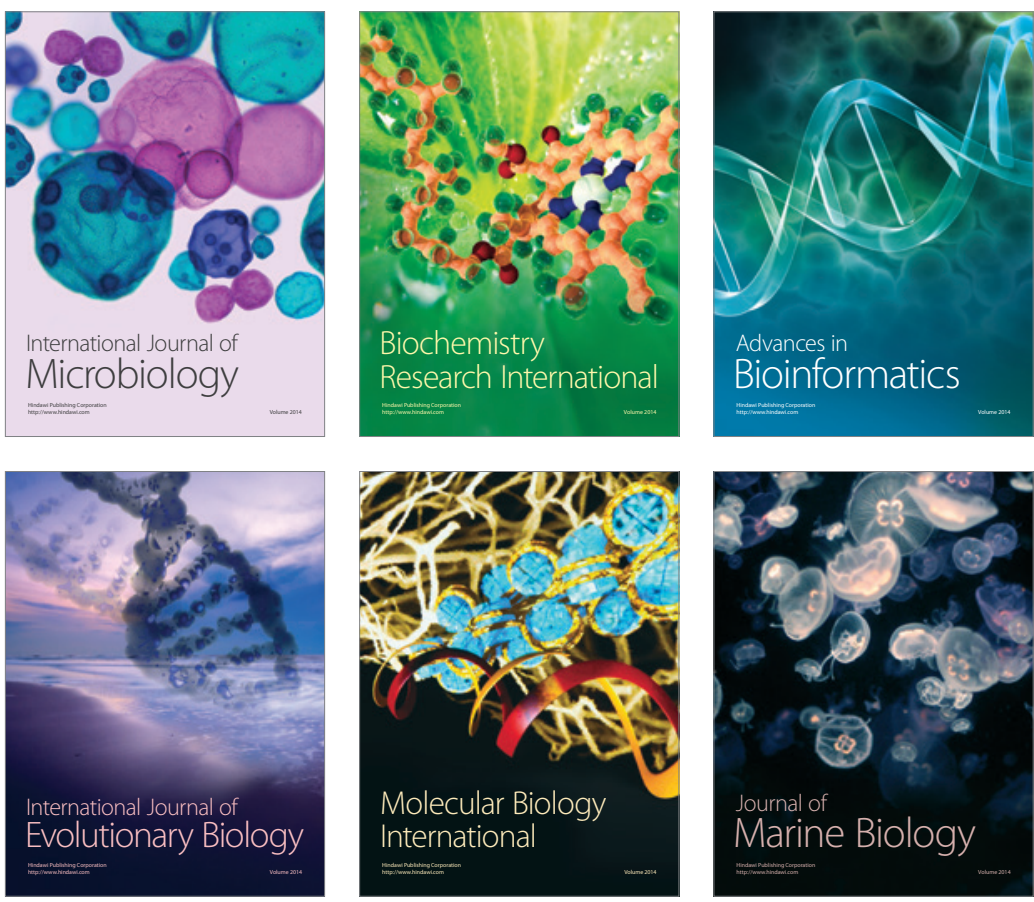